\title{
Usual Closed Birth Interval versus Most Recent Closed Birth Interval
}

\author{
Anup Kumar ${ }^{1 *}$ and R. C. Yadava ${ }^{2}$ \\ ${ }^{1}$ Department of Statistics, University of Allahabad \\ ${ }^{2}$ Department of Statistics \& DST-CIMS, Banaras Hindu University
}

\begin{abstract}
Normally, one may think that the distribution of closed birth interval of any specific order may be the same as the distribution of most recent closed birth interval of the same order. But it is not true. Here the distinction between the distribution of a specific order of usual closed birth interval and most recent closed birth interval of the same order is examined. In this context, firstly we demonstrate the distinction between the most recent closed birth interval and usual closed birth interval empirically by considering a real data set. Further, the distinction between these distributions is demonstrated theoretically, by taking certain hypothetical values of fertility parameters involved in the stochastic model proposed for the purpose.
\end{abstract}

Key words:

\section{Introduction}

The data on birth intervals can be obtained in quite different ways under different sampling frames, and hence appropriate techniques are needed for proper analysis of such data, otherwise one may draw misleading and invalid conclusions [see Fisher (1934); Rao (1965); Srinivasan (1967); Wolfers (1968); Sheps et al. (1970); Sheps and Menken (1972, 1973); Singh et al. (1979); Srinivasan (1980); Yadava and Sharma (2004) and others]. For example, a female having given three births from marriage to end of reproductive span or survey date, has three birth intervals $(i)$ interval from marriage to first birth, (ii) interval from first to second birth and (iii) interval from second to third birth. In general, the interval between $i^{t h}$ and $(i+1)^{\text {th }}$ births is normally called as closed birth interval (CBI) of order $i$ or $i^{\text {th }}$ order CBI. In retrospective fertility surveys, the CBI of $i^{\text {th }}$ order is defined only for those females who have given at least $(i+1)$ births in the given marital duration. A CBI of any specific order is normally ascertained for all those women who ever experienced that interval, but for each female there will be a last CBI which is a parityspecific most recent CBI before the date of survey. To make a distinction between the two, the earlier is termed as usual CBI while the latter as most recent CBI. Thus the $i^{\text {th }}$ order usual CBI is defined for those females who have given at least $(i+1)$ births while $i^{\text {th }}$ order most recent CBI is defined for those females who have given exactly $(i+1)$ births. The most recent CBI is

\footnotetext{
${ }^{*}$ Corresponding author.
} 
relatively easy to be ascertained and refers closely to the current fertility of women in the population under study. The distribution of most recent CBI is quite sensitive to small changes in fertility and robust with respect to non-fertility parameters [see Sehgal (1971)]. The collection of birth interval data in underdeveloped countries suffers from many non-sampling errors; especially recall lapse on the part of respondents. These errors, which are sometimes serious, often vitiate the data if the duration of time elapsed since the occurrence of the event is large. Consequently, the data on most recent CBI seem to be less affected by such memory biases and, they are likely to be more reliable for the analysis of fertility changes among married women compared to usual CBI.

Usually, one may think that the distribution of CBI of any specific order may be the same as the distribution of most recent CBI of the same order. But it is not true. Sheps and Menken (1972) have remarked that for a given age (or marital duration), the mean of most recent CBI is somewhat higher than the other CBI based on the cohort approach, as this interval usually tends to select larger values more frequently resulting in higher mean. Sharma (2004) has also considered the distinction between the most recent CBI and usual CBI. He assumes a stationary population of females, where specific order of births are uniformly distributed over time, and demonstrated that, if the population of females is homogeneous with respect to fertility parameters (conception rate and post-partum amenorrhoea (PPA) period), then there is no difference between the distribution of usual CBI and most recent CBI. Whereas, if females are heterogeneous with respect to the fertility parameters, then the two distributions are distinct, and the mean of most recent CBI is somewhat larger than the mean of usual CBI of that order.

Some general distributions for the most recent CBI have been derived by Poole (1973) and Sheps and Menken (1972), Sheps and Menken (1973). Sheps and Menken (1973) have derived results regarding the most recent $\mathrm{CBI}$ considering general distributions between two events (births). Based on these results, they have given their remarks regarding most recent CBI, wherein, one has to assume some specific forms of distribution functions of various components of a birth interval. Singh et al. (1988) derived probability models for the most recent CBI considering specific distributions for various components of CBI. Later, Pandey et al. (1998) extended the above model accounting for the non-exposure period in the beginning of the reproductive life, caused due to adolescent sterility and temporary separation between the partners. Both have illustrated their models by applying them on an observed set of data. However, both the models do not attempt to study the distinction between the usual CBI and the most recent CBI. Abul-ata (1987) has derived expressions for the distributions of usual CBI and most recent CBI for specific parity with fixed marital duration by considering the interval between consecutive births as exponentially distributed. This assumption does not seem to be appropriate in the context of birth interval studies, because the timing between consecutive births not only includes the menstruating interval but also the gestation period and PPA period; which essentially cannot be ignored as gestation period itself is around 9 months and average PPA may be somewhat larger than 6 months.

Here the distinction between the distributions of various orders of usual CBI and most recent CBI under the condition of fixed marital duration is examined. In this context, firstly, the distinction between most recent $\mathrm{CBI}$ and usual $\mathrm{CBI}$ is demonstrated, empirically, by considering 
a real data set of NFHS-3. In Section 2, the results, using the NFHS-3 (2005-06) data for Uttar Pradesh (U.P.), are demonstrated. But the results for other states can also be demonstrated on similar lines. Further in Section 3, the distinction between distributions of usual CBI and most recent $\mathrm{CBI}$ for specific parity of fixed marital duration is theoretically demonstrated by taking certain hypothetical values of the fertility parameters.

\section{Empirical Data on Closed Birth Intervals and Most Recent Closed Birth Interval for Specified Marital Duration}

The means of usual CBI of different order and the mean of most recent CBI for females of specified marital durations are presented using the data of NFHS-3 (U.P.). The considered marital durations are 5-9, 10-14, 15-19, 20-24, 25-29, 30+ years. At last, a table considering all marital durations is also presented (see Tables 1-7).

For a given marital duration and fixed parity, the mean of the most recent closed birth interval is significantly larger than the means of other usual CBI. This particular result is common in every table (see Tables 1-7). The differences in the two means are somewhat between 3-6 months, which cannot be ignored on the basis of sampling or non-sampling errors in the data. For example, in Table 7 there are 1006 females who have given 5 births and among them the means of first, second and third order CBI are 28.46, 30.29 and 30.02 months respectively, while for the fourth order it is 35.50 months. Thus the mean of most recent CBI is around 5 months larger than the other means. Certainly, the two distributions of corresponding birth intervals are different even though there components are the same. These results reveal that the mean of most recent CBI is significantly larger than usual CBI as remarked by Sheps and Menken (1972).

Another issue of the importance of sampling frame for CBI is also explained here. From the Tables $1-7$, it is very much evident that the mean birth intervals for different order vary to a large extent according to marital duration as well as order of birth. For example, if the marital duration is 5-9 years, then females giving exactly two births in this marital duration have the mean CBI between first and second births as 34.24 months, while this mean for marital duration 10-14 years and 15-19 years becomes 44.15 and 46.08 months respectively. Similarly, for the females giving exactly three births in marital duration 10-14 years, the mean interval between first and second births is 31.98 months, while this average is 35.29 months for marital duration 15-19 years. Similar observations are found for most of the cases in the tables considered for illustration. The question arises; whether one can have better idea of the mean CBI for different orders of births from the above tables. In the bottom of each table, mean CBI for different orders are also given for different marital durations. In this context, the first order CBI for marital duration 5-9 years (i.e. interval between first and second births) is 29.04 months. The question is whether this can be considered as true mean of first order CBI. The answer is no, because normally all females do not have sufficient time to have at least two births in this marital duration, and this CBI is defined only for those females who have given at least two births in this interval. Thus, larger birth intervals are excluded from the study resulting in lower observed mean. This is precisely the truncation effect as discussed in Sheps et al. (1970). Hence, the mean 29.04 months may be 
considered as an under estimate of mean for CBI of first order. However, this mean for the marital duration 10-14 years is 30.93 months while for marital durations 15-19 years, 20-24 years, 2529 years and $30+$ years, the means are $31.84,32.61,33.77$ and 34.41 months, respectively. It is quite reasonable to assume that the probability of having at least two births in the marital duration 10-14 years and onwards is almost one, and these means can be considered as reasonable estimates of the means of the first order CBI. Thus, the interpretation of mean birth interval should be done very cautiously, otherwise one may have incorrect and misleading conclusions.

Table 1: Various order mean closed birth intervals (in months) for females giving different number of births in marital duration (5-9) years(U.P. NFHS-3 data)

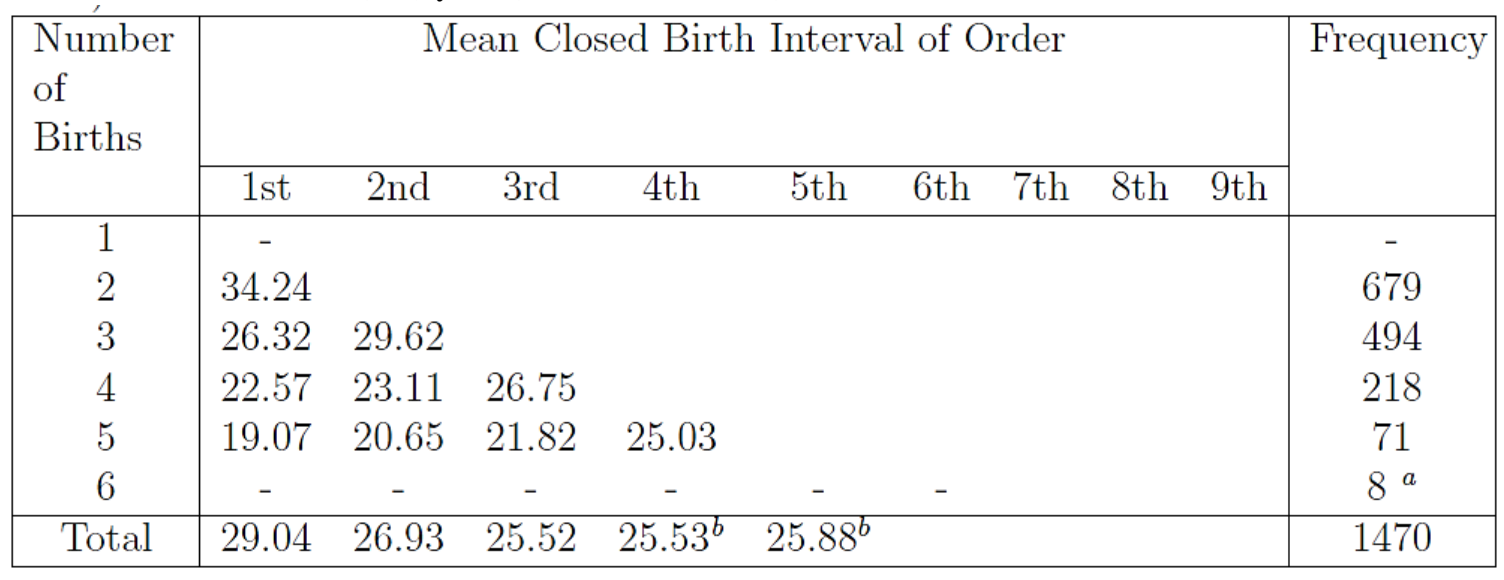

${ }^{a}$ very less number of observations

${ }^{b}$ mean based on very less number of observations 
Table 2: Various order mean closed birth intervals (in months) for females giving different number of births in marital duration (10-14) years(U.P. NFHS-3 data)

\begin{tabular}{|c|c|c|c|c|c|c|c|c|c|c|}
\hline \multirow{2}{*}{$\begin{array}{l}\text { Number } \\
\text { of } \\
\text { Births }\end{array}$} & \multicolumn{9}{|c|}{ Mean Closed Birth Interval of Order } & \multirow[t]{2}{*}{ Frequency } \\
\hline & 1 st & 2nd & 3rd & 4 th & 5 th & 6 th & 7 th & 8 th & 9 th & \\
\hline 1 & - & & & & & & & & & - \\
\hline 2 & 44.15 & & & & & & & & & 294 \\
\hline 3 & 31.98 & 36.88 & & & & & & & & 374 \\
\hline 4 & 28.94 & 29.42 & 34.58 & & & & & & & 361 \\
\hline 5 & 24.28 & 26.40 & 26.49 & 32.82 & & & & & & 260 \\
\hline 6 & 22.13 & 23.29 & 22.79 & 24.37 & 28.46 & & & & & 130 \\
\hline 7 & 20.52 & 20.98 & 19.51 & 23.04 & 22.15 & 26.51 & & & & 55 \\
\hline 8 & - & - & - & - & - & - & - & - & & $10^{a}$ \\
\hline 9 & - & - & - & - & - & - & - & - & & $2^{a}$ \\
\hline Total & 30.93 & 29.91 & 28.91 & 28.92 & 25.93 & $25.68^{b}$ & $20.58^{b}$ & $17.50^{b}$ & & 1486 \\
\hline
\end{tabular}

${ }^{a}$ very less number of observations

${ }^{b}$ mean based on very less number of observations

Table 3: Various order mean closed birth intervals (in months) for females giving different number of births in marital duration (15-19) years(U.P. NFHS-3 data)

\begin{tabular}{|c|c|c|c|c|c|c|c|c|c|c|}
\hline \multirow{2}{*}{$\begin{array}{l}\text { Number } \\
\text { of } \\
\text { Births }\end{array}$} & \multicolumn{9}{|c|}{ Mean Closed Birth Interval of Order } & \multirow[t]{2}{*}{ Frequency } \\
\hline & 1st & 2nd & 3rd & 4th & 5 th & 6 th & 7 th & 8 th & 9 th & \\
\hline 1 & - & & & & & & & & & - \\
\hline 2 & 46.08 & & & & & & & & & 175 \\
\hline 3 & 35.29 & 43.40 & & & & & & & & 271 \\
\hline 4 & 30.13 & 32.95 & 38.44 & & & & & & & 279 \\
\hline 5 & 28.32 & 31.01 & 29.26 & 36.94 & & & & & & 268 \\
\hline 6 & 29.22 & 25.79 & 27.89 & 28.65 & 32.40 & & & & & 223 \\
\hline 7 & 26.93 & 26.36 & 23.35 & 23.18 & 26.83 & 30.19 & & & & 111 \\
\hline 8 & 23.24 & 22.97 & 24.8 & 22.55 & 23.74 & 24.23 & 33.24 & & & 74 \\
\hline 9 & - & - & - & - & - & - & - & - & & $18^{a}$ \\
\hline 10 & - & - & - & - & - & - & - & - & - & $14^{a}$ \\
\hline Total & 31.84 & 32.03 & 30.35 & 29.89 & 28.77 & 26.89 & $30.46^{b}$ & $23.78^{b}$ & $24.57^{b}$ & 1433 \\
\hline
\end{tabular}

${ }^{a}$ very less number of observations

${ }^{b}$ mean based on very less number of observations 
Table 4: Various order mean closed birth intervals (in months) for females giving different number of births in marital duration (20-24) years(U.P. NFHS-3 data)

\begin{tabular}{|c|c|c|c|c|c|c|c|c|c|c|}
\hline \multirow{2}{*}{$\begin{array}{l}\text { Number } \\
\text { of } \\
\text { Births }\end{array}$} & \multicolumn{9}{|c|}{ Mean Closed Birth Interval of Order } & \multirow[t]{2}{*}{ Frequency } \\
\hline & 1st & 2nd & 3rd & 4 th & 5 th & 6 th & 7 th & 8 th & 9th & \\
\hline 1 & - & & & & & & & & & - \\
\hline 2 & 45.20 & & & & & & & & & 138 \\
\hline 3 & 37.03 & 48.47 & & & & & & & & 223 \\
\hline 4 & 33.43 & 31.31 & 39.41 & & & & & & & 196 \\
\hline 5 & 30.25 & 30.52 & 33.45 & 37.72 & & & & & & 206 \\
\hline 6 & 30.75 & 32.11 & 30.78 & 30.84 & 37.74 & & & & & 183 \\
\hline 7 & 28.78 & 26.96 & 27.22 & 25.56 & 28.73 & 36.37 & & & & 145 \\
\hline 8 & 25.33 & 26.21 & 26.08 & 24.98 & 26.47 & 25.49 & 35.45 & & & 87 \\
\hline 9 & 23.46 & 24.53 & 22.61 & 22.76 & 23.82 & 23.70 & 25.29 & 36.74 & & 66 \\
\hline 10 & 24.42 & 25.06 & 21.55 & 23.74 & 21.97 & 25.81 & 26.97 & 23.94 & 28.39 & 31 \\
\hline Total & 32.61 & 33.15 & 31.31 & 29.98 & 30.52 & 29.95 & 30.37 & 32.64 & 28.39 & 1275 \\
\hline
\end{tabular}

Table 5: Various order mean closed birth intervals (in months) for females giving different number of births in marital duration (25-29) years(U.P. NFHS-3 data)

\begin{tabular}{|c|c|c|c|c|c|c|c|c|c|c|}
\hline \multirow{2}{*}{$\begin{array}{l}\text { Number } \\
\text { of } \\
\text { Births }\end{array}$} & \multicolumn{9}{|c|}{ Mean Closed Birth Interval of Order } & \multirow[t]{2}{*}{ Frequency } \\
\hline & 1st & 2nd & 3rd & 4 th & 5 th & 6 th & 7 th & 8 th & 9 th & \\
\hline 1 & - & & & & & & & & & - \\
\hline 2 & 52.82 & & & & & & & & & 65 \\
\hline 3 & 38.16 & 46.87 & & & & & & & & 116 \\
\hline 4 & 34.39 & 33.74 & 37.79 & & & & & & & 136 \\
\hline 5 & 32.16 & 35.67 & 33.76 & 36.09 & & & & & & 138 \\
\hline 6 & 32.08 & 34.57 & 30.71 & 32.35 & 36.81 & & & & & 150 \\
\hline 7 & 28.79 & 30.17 & 29.01 & 33.10 & 30.60 & 33.02 & & & & 105 \\
\hline 8 & 29.12 & 30.10 & 28.60 & 29.29 & 28.76 & 29.94 & 34.93 & & & 101 \\
\hline 9 & 29.91 & 29.27 & 27.73 & 27.39 & 24.64 & 25.25 & 27.06 & 30.75 & & 64 \\
\hline 10 & 27.25 & 25.76 & 26.12 & 33.18 & 28.82 & 26.71 & 24.00 & 24.76 & 29.94 & 17 \\
\hline Total & 33.77 & 34.64 & 31.72 & 32.31 & 31.36 & 29.82 & 31.14 & 29.49 & 29.94 & 892 \\
\hline
\end{tabular}


Table 6: Various order mean closed birth intervals (in months) for females giving different number of births in marital duration (30+) years(U.P. NFHS-3 data)

\begin{tabular}{|c|c|c|c|c|c|c|c|c|c|c|}
\hline \multirow{2}{*}{$\begin{array}{l}\text { Number } \\
\text { of } \\
\text { Births }\end{array}$} & \multicolumn{9}{|c|}{ Mean Closed Birth Interval of Order } & \multirow[t]{2}{*}{ Frequency } \\
\hline & 1st & 2nd & 3rd & 4 th & 5 th & 6 th & 7 th & 8 th & 9 th & \\
\hline 1 & - & & & & & & & & & - \\
\hline 2 & 46.56 & & & & & & & & & 18 \\
\hline 3 & 39.40 & 51.45 & & & & & & & & 49 \\
\hline 4 & 35.38 & 41.56 & 39.45 & & & & & & & 78 \\
\hline 5 & 42.79 & 41.59 & 37.59 & 41.76 & & & & & & 63 \\
\hline 6 & 32.40 & 29.59 & 37.93 & 32.33 & 39.00 & & & & & 58 \\
\hline 7 & 27.81 & 31.8 & 35.76 & 31.43 & 30.54 & 33.43 & & & & 54 \\
\hline 8 & 29.06 & 29.17 & 30.11 & 34.17 & 35.89 & 27.89 & 44.22 & & & 36 \\
\hline 9 & 28.19 & 25.16 & 30.53 & 25.16 & 30.13 & 30.19 & 30.09 & 31.19 & & 32 \\
\hline 10 & 24.81 & 24.86 & 22.29 & 26.33 & 27.00 & 29.95 & 26.38 & 27.86 & 34.19 & 21 \\
\hline Total & 34.41 & 36.30 & 35.39 & 33.30 & 33.5 & 30.79 & 34.93 & 29.87 & 34.19 & 409 \\
\hline
\end{tabular}

Table 7: Various order mean closed birth intervals (in months) for females giving different number of births in all marital durations (U.P. NFHS-3 data)

\begin{tabular}{|c|c|c|c|c|c|c|c|c|c|c|}
\hline \multirow{2}{*}{$\begin{array}{l}\text { Number } \\
\text { of } \\
\text { Births }\end{array}$} & \multicolumn{9}{|c|}{ Mean Closed Birth Interval of Order } & \multirow[t]{2}{*}{ Frequency } \\
\hline & 1st & 2nd & 3rd & 4 th & 5 th & 6 th & 7th & 8 th & 9 th & \\
\hline 1 & - & & & & & & & & & - \\
\hline 2 & 37.16 & & & & & & & & & 1621 \\
\hline 3 & 31.93 & 38.22 & & & & & & & & 1558 \\
\hline 4 & 29.76 & 30.59 & 35.44 & & & & & & & 1270 \\
\hline 5 & 28.46 & 30.29 & 30.02 & 35.38 & & & & & & 1006 \\
\hline 6 & 29.1 & 28.89 & 29.02 & 29.48 & 34.34 & & & & & 752 \\
\hline 7 & 27.27 & 27.39 & 26.79 & 27.06 & 28.14 & 32.84 & & & & 470 \\
\hline 8 & 26.40 & 26.72 & 26.87 & 26.79 & 27.29 & 26.86 & 35.33 & & & 308 \\
\hline 9 & 26.81 & 25.93 & 26.07 & 24.60 & 25.04 & 24.97 & 26.39 & 32.51 & & 182 \\
\hline 10 & 24.74 & 24.24 & 22.66 & 25.27 & 24.51 & 26.59 & 26.00 & 24.31 & 29.53 & 83 \\
\hline Total & 31.19 & 31.69 & 30.58 & 30.45 & 30.10 & 29.20 & 31.13 & 29.94 & 29.53 & 7250 \\
\hline
\end{tabular}




\section{Stochastic Models for Usual Closed Birth Interval and Most Recent Closed Birth Interval}

Certain models are derived to demonstrate the difference between usual CBI and most recent CBI for specified marital duration. Abul-ata (1987) has also derived such results. But in his approach, the distribution between consecutive births has been considered to be exponentially distributed implying that after a birth there is a possibility of another birth, just after it. This is totally unrealistic and can be viewed only as a theoretical result. A more realistic situation has been considered, where after a birth the next conception may take place after the non-susceptible period $(\mathrm{h})$, which is the sum of gestation period and PPA period. If one to one correspondence between conception and birth is assumed, then the number of conceptions is equivalent to number of births. Similarly, the duration of $i^{\text {th }}$ order CBI (duration between $i^{\text {th }}$ and $(i+1)^{\text {th }}$ births), $i \geq 1$, is same as the duration between $i^{\text {th }}$ and $(i+1)^{\text {th }}$ conceptions. So without loss of generality, we have derived the results for duration between consecutive conceptions called closed conception interval rather than between consecutive births. Before deriving the results, a brief description of various notations used (which are similar to the notations used in Abul-ata (1987)), is given.

\section{Notations}

$X_{0} \quad$ is the random length of the $0^{\text {th }}$ order conception time i.e. interval from marriage to first conception.

$X_{i} \quad$ is the random length of the interval from the $(i)^{t h}$ conception to the $(i+1)^{t h}$ conception when the potential duration of a fecund marriage is assumed to be infinnite; i.e., every married women will experience the interval $X_{i}$ with certainty. The density and distribution functions of variable $X_{i}$ are $f_{i}(\mathrm{x})$ and $F_{i}(\mathrm{x})$ respectively. $i \geq 0$

$S_{i} \quad$ is the total waiting time from marriage till the occurrence of $(i+1)^{t h}$ conception, $S_{i}=\sum_{j=0}^{i} X_{j}$ The density and distribution function of variable $S_{i}$ are $g_{i}(x)$ and $G_{i}(x)$ respectively.

$T \quad$ is the exposure time for conceptions measured at survey date. $\mathrm{T}=T^{\prime}-g$, where $T^{\prime}$ is the duration of marriage measured at survey date.

$P_{i}(T)$ is $\operatorname{Pr}[C(0, T)=i]$, where $C(0, T)$ is the counting process that counts the number of conceptions in $(0, T)$.

$X_{i}(T)$ is the random length of the interval from the $(i)^{t h}$ conception to the $(i+1)^{t h}$ conception ; i.e. interval between $(i)^{t h}$ conception to the $(i+1)^{t h}$ conception conditional on length $\mathrm{T}$. The density and distribution functions of variable $X_{i}(X, T)$ are $h_{i}(X, T)$ and $H_{i}(X, T)$ respectively.

$X_{i}^{l}(T)$ is the random length of the last closed conception interval conditional on length $\mathrm{T}$ where $(i+1)^{t h}$ conception happens to be the last conception in $(0, T)$. The density and distribution functions of variable $X_{i}^{l}(T)$ are $h_{i}^{l}(X, T)$ and $H_{i}^{l}(X, T)$ respectively. 


\section{Closed Conception Interval}

$X_{i}(T)$ is a random variable denoting the length of the interval (if exists) be-tween the $(i)^{t h}$ conception and the $(i+1)^{t h}$ conception, where the conception process is being truncated at $T$.

$$
X_{i}(T) \leq T
$$

$H_{i}(X, T)$ is given as

$$
\begin{aligned}
H_{i}(x, T) & =P_{r}\left(X_{i}(T) \leq x\right) \\
& =P_{r}\left(X_{i} \leq x \mid S_{i+1} \leq T\right) \\
& =\frac{P_{r}\left(X_{i} \leq x \mid S_{i+1} \leq T\right)}{G_{i+1}(T)}
\end{aligned}
$$

now

$$
\begin{aligned}
P_{r}\left(X_{i} \leq x \cap S_{i+1} \leq T\right) & =\int_{0}^{x} P_{r}\left(S_{i+1} \leq T \mid X_{i}=y\right) f_{i}(y) d y \\
& =\int_{0}^{x} P_{r}\left(S_{i} \leq T-y\right) f_{i}(y) d y .
\end{aligned}
$$

Thus, the distribution function for $X_{i}(T)$ is

$$
H_{i}(x, T)=\frac{\int_{0}^{x} G_{i}(T-y) f_{i}(y) d y}{G_{i+1}(T)}, x \leq T
$$

and the density function of $X_{i}(T)$ is

$$
h_{i}(x, T)=\frac{\mathrm{d}}{\mathrm{dx}} H_{i}(T)=\frac{G_{i}(T-x) f_{i}(x)}{G_{i+1}(T)}, \quad x \leq T .
$$

\section{Retrospective Last Closed Conception Interval}

The distribution function of $X_{i}^{l}(T)$, is de ned as,

$$
\begin{aligned}
H_{i}(x, T) & =P_{r}\left(X_{i}^{l}(T) \leq x\right) \\
& =P_{r}\left(X_{i} \leq x \mid \operatorname{exactly}(i+1) \text { conceptions in }(0, T)\right) \\
& =\frac{P_{r}\left(X_{i} \leq x \cap C(0, T)=1+\mathrm{i}\right)}{P_{i+1}(T)} \\
& =\frac{\left.\int_{0}^{x} P_{r} C(0, T)=i+1 \mid X_{i}=y\right) f_{i}(y) d y}{P_{i+1}(T)} \\
& =\frac{\left.\int_{0}^{x} P_{r} C(0, T-y)=i+1 \mid X_{i}=y\right) f_{i}(y) d y}{P_{i+1}(T)},
\end{aligned}
$$

and 


$$
H_{i}^{l}(x, T)=\frac{\int_{0}^{x} P_{r}(T-y) f_{i}(y) d y}{P_{i+1}(T)}
$$

Thus,

$$
h_{i}^{l}(x, T)=\frac{P_{i}(T-x) f_{i}(x)}{P_{i+1}(T)}, i \geq 1, x \leq T .
$$

Here, general stochastic models have been derived for the usual closed conception interval and the most recent conception interval. But for the analysis and comparison, some specific forms for $f_{i}(x)$ has been assumed. As it has been mentioned earlier that Abul-ata (1987) considered $f_{i}(x)$ to follow Exponential distribution

$$
f_{i}(x)=\lambda e^{-\lambda x}, \lambda>0, x>0, i \geq 0,
$$

and showed that

$$
\int_{0}^{t} g_{i+1}(s) d s=1-\sum_{j=0}^{i} e^{-\lambda T} \frac{(\lambda T)^{j}}{j !},
$$

and the distribution for closed conception interval is given as;

$$
h_{i}(x, T)=\frac{\lambda e^{-\lambda x}-\lambda e^{-\lambda T} \sum_{j=0}^{i-1} \frac{\lambda^{j+1}}{j !}}{1-\sum_{j=0}^{i} e^{-\lambda T} \frac{(\lambda T)^{j}}{j !}} \text { for } i \geq 1, x \leq T, \lambda \geq 0,
$$

and expectation of $X_{i}(T)$;

$$
E\left(X_{i}(T)\right)=\frac{\lambda^{-1}-e^{-\lambda T}\left(T+\lambda^{-1}+\lambda^{-1} \sum_{j=0}^{i-1} \frac{(\lambda T)^{j+2}}{(j+2) !}\right)}{1-\sum_{j=0}^{i} e^{-\lambda T^{(\lambda T)^{j}}}}, \text { for } i \geq 1
$$

Similarly, the distribution of most recent closed conception interval is given as

$$
\begin{aligned}
h_{i}^{l}(x, T) & =\frac{\frac{\left(\lambda(T-x)^{i}\right) e^{-\lambda(T-x)}}{i !}}{\frac{(i+1)(T-x)^{i}}{T^{i+1}}} \lambda e^{-\lambda x} \\
& =\frac{(i+1)(T-x)^{i}}{T^{i+1}}, i \geq 1, x \leq T .
\end{aligned}
$$

The above distribution is independent of the parameter $\lambda$, i.e., conception rate.

The corresponding expression for mean is given as

$$
E\left(X_{i}^{l}(T)\right)=\frac{T}{i+2}, i \geq 1 .
$$

Already, the serious limitations of this model have been mentioned. Now, the distributions of usual conception interval and most recent closed conception interval are derived based on the following assumptions:

- $\quad$ The female has led a married life throughout the period $(0, T)$.

- The time interval of the first conception after marriage follows an exponential distribution with probability density function (p.d.f.)

$$
f_{0}(x)=\lambda e^{-\lambda x}, \quad x \geq 0, \lambda \geq 0 .
$$


We call the parameter $\lambda$, the conception rate.

- The duration between $(i)^{t h}$ and $(i+1)^{t h}$ conceptions follows a displaced exponential distribution with p.d.f.

$$
f_{i}(x)=f(x)=\lambda e^{-\lambda(x-h)}, \quad x \geq h, \lambda \geq 0, i \geq 1 .
$$

This assumption implies that after a conception there is no possibility of another conception for a period $h$, where $h$ is the sum of duration of pregnancy $(\mathrm{g})$ and postpartum amenorrhoea(PPA) associated with a birth (or conception).

- Every conception results in a live birth.

Note: Since, the distribution of $X_{0}$ is di erent than the distribution of $X_{i}, i \geq 1$, hence the analysis is confined for closed conception interval of order $i \geq 1$.

These assumptions have also been used by many authors in deriving the models for either the number of births to a female or the birth intervals, and due to flexibility of the probability expressions, these models can easily be applied to data, relating to variety of situations [see Singh (1968); Singh et al. (1971, 1974) and others].

Under the above assumptions

$$
\begin{aligned}
& P\left(X_{0} \leq x\right)=1-e^{-\lambda x}, \quad x \geq 0 \\
& P\left(X_{i} \leq x\right)=1-e^{-\lambda(x-h)}, x \geq h, \text { for } i=1,2,3, \ldots
\end{aligned}
$$

and the sequence $\left\{X_{0}, X_{1}, X_{2}, \ldots\right\}$ are mutually independently distributed random variables. $G_{i+1}(T)$ is the distribution function of time for $(i+1)^{t h}$ conception from marriage, which is the probability of at least $(i+1)$ conceptions in $(0, T)$. Then the expression for $G_{i+1}(T)=P\left(X_{0}+\right.$ $\left.X_{1}+X_{2}+\cdots+X_{i} \leq T\right)$ is obtained [see Singh (1968)] as

and

$$
G_{i+1}(T)=\left\{\begin{array}{l}
1-e^{-\lambda(T-i h)} \sum_{j=0}^{i} \frac{\lambda^{j}(T-i h)^{j}}{j !}, T \geq i h \\
0, \quad T<i h,
\end{array}\right.
$$

$P_{i+1}(T)\left\{\begin{array}{l}G_{i+1}(T)-G_{i+2}(T), \text { for } T>(i-1) h=e^{-\lambda(T-(i+1) h)} \sum_{j=0}^{i+1} \frac{\lambda^{j}(T-(i+1) h)^{j}}{j !}-e^{-\lambda(T-i h)} \sum_{j=0}^{i} \frac{\lambda^{j}(T-i h)^{j}}{j !} \\ G_{i+1}(T), \text { for } i h<T<(i-1) h=1-e^{-\lambda(T-i h)} \sum_{j=0}^{i} \frac{\lambda^{j}(T-i h)^{j}}{j !} \\ 0, \quad \text { for } T<i h .\end{array}\right.$

Note: From the above, it is clear that for specified values of $T$ and $h$, there will be a maximum possible value of $i$ for which $i^{\text {th }}$ order closed conception interval is defined.

Now, substituting the values of $G_{i+1}(T)$ and $G_{i}(T-x)$ in Equation (2), the density function for $(i)^{t h}$ order usual closed conception interval for $i \geq 1$. is given as

$$
h_{i}(x, T)=\left\{\begin{array}{l}
\frac{G_{i}(T-x)}{G_{i+1}(T)} f(x), \quad h<x<T-(i-1) \\
0, \quad \text { otherwise. }
\end{array}\right.
$$


The expected value of $X_{i}(T)$, is given as under

$$
\begin{aligned}
& E\left(X_{i}(T)\right)=\frac{1}{G_{i+1}(T)} \int_{h}^{T-(i-1) h} x G_{i}(T-x) f(x) d x \\
& =\frac{1}{G_{i+1}(T)} \int_{h}^{T-(i-1) h} \lambda e^{-\lambda(x-h)}\left(1-e^{-\lambda(T-(i-1) h-x)} \sum_{j=0}^{i=1} \frac{\lambda^{j}(T-(i-1) h-x)^{j}}{j !}\right) x d x \\
& =\frac{1}{G_{i+1}(T)}\left(\frac{\lambda h+1}{\lambda}-\frac{\lambda T^{\prime}}{\lambda} e^{-\lambda\left(T^{\prime}-h\right)}\right. \\
& \left.\quad-\lambda e^{-\lambda\left(T^{\prime}-h\right)}\left(\sum_{j=0}^{i-1}\left(h \frac{\lambda^{j}}{(j+1) !}\left(T^{\prime}-h\right)^{j+1}+\frac{\lambda^{j}}{(j+2) !}\left(T^{\prime}-h\right)^{j+2}\right)\right)\right),
\end{aligned}
$$

where $T^{\prime}=T-(i-1) h$. And the expression for the density of most recent closed conception interval is given as under

$$
h_{i}^{l}(x, T)\left\{\begin{array}{l}
\frac{P_{i}(T-x)}{P_{i+1}(T)} f(x), h<x<T-i h \\
\frac{G_{i}(T-x)}{P_{i+1}(T)} f(x), T-i h<x<T-(i-1) h \\
\text { because for } T-i h<x<T-(i-1) h, G_{(i+1)}(T-x)=0 \\
0, \text { otherwise. }
\end{array}\right.
$$

The corresponding expression for the mean of most recent closed conception interval is given as

$$
\begin{gathered}
E\left(X_{i}^{l}(T)\right)=\frac{1}{P_{i+1(T)}}\left(\int_{h}^{T^{\prime \prime}} x P_{i}(T-x) f(x) d x+\int_{T^{\prime \prime}}^{T^{\prime}} x G_{i}(T-x) f(x) d x\right) \\
=\frac{1}{P_{i+1(T)}}\left(\int _ { h } ^ { T ^ { \prime \prime } } \left(e^{-\lambda(T-x-i h)} \sum_{j=0}^{i} \frac{\lambda^{j}(T-x-i h)^{j}}{j !}\right.\right. \\
\left.-e^{-\lambda(T-x-(i-1) h)} \sum_{j=0}^{i-1} \frac{\lambda^{j}(T-x-(i-1) h)^{j}}{j !}\right) \lambda e^{-\lambda(x-h)} x d x \\
\left.+\int_{T^{\prime \prime}}^{T^{\prime}}\left(1-e^{-\lambda(T-x-(i-1) h)} \sum_{j=0}^{i-1} \frac{\lambda^{j}(T-x-(i-1) h)^{j}}{j !}\right) \lambda e^{-\lambda(x-h)} x d x\right),
\end{gathered}
$$

where $T^{\prime}=T-(i-1) h$ and $T^{\prime \prime}=T-i h$

The above Expression (12) can be written as

$$
E\left(X_{i}^{l}(T)\right)=\frac{1}{P_{i+1}(T)}\left(\phi_{1}-\phi_{2}+\phi_{3}-\phi_{4}\right),
$$


where

$$
\begin{aligned}
& \phi_{1}=\int_{h}^{T^{\prime \prime}}\left(e^{-\lambda(T-x-i h)} \sum_{j=0}^{i} \frac{\lambda^{j}(T-x-i h)^{j}}{j !}\right) \lambda e^{-\lambda(x-h)} x d x \\
& =\lambda e^{-\lambda\left(T^{\prime \prime}-h\right)}\left[\sum_{j=0}^{i} \frac{\lambda^{j}}{(j+2) !}\left[h(j+2)\left(T^{\prime \prime}-h\right)^{(j+2)}\right]\right] \\
& \phi_{2}=\int_{h}^{T^{\prime \prime}}\left(e^{-\lambda(T-x-(i-1) h)} \sum_{j=0}^{i} \frac{\lambda^{j}(T-x-(i-1) h)^{j}}{j !}\right) \lambda e^{-\lambda(x-h)} x d x \\
& =\lambda e^{-\lambda T^{\prime \prime}}\left[\sum_{j=0}^{i-1} \frac{\lambda^{j}}{(j+2) !}\left[h(j+2) T^{\prime \prime(j+1)}-T^{\prime \prime}(j+2) h^{(j+1)}+T^{\prime \prime(j+2)}-h^{(j+2)}\right]\right] \\
& \phi_{3}=\int_{T^{\prime \prime}}^{T^{\prime}} x \lambda e^{-\lambda(x-h)}=e^{-\lambda\left(T^{\prime \prime}-h\right)}\left(T^{\prime \prime}+\frac{1}{\lambda}\right)-e^{-\left(T^{\prime}-h\right)},
\end{aligned}
$$

and

$$
\begin{aligned}
\phi_{4} & =\int_{h}^{T^{\prime \prime}}\left(e^{-\lambda(T-x-(i-1) h)} \sum_{j=0}^{i-1} \frac{\lambda^{j}(T-x-(i-1) h)^{j}}{j !}\right) \lambda e^{-\lambda(x-h)} x d x \\
& =\lambda e^{-\lambda T^{\prime \prime}}\left(\sum_{j=0}^{i-1} \frac{\lambda^{j}}{(j+2) !}\left[(j+2) T^{\prime \prime} h^{(j+1)}+h^{(j+2)}\right]\right) .
\end{aligned}
$$

Substituting the values of $\phi_{1}, \phi_{2}, \phi_{3}$ and $\phi_{4}$ [from Expressions (14), (15), (16) and (17)] in Expression (13), the value of $E\left(X_{i}^{l}(T)\right)$ can be obtained.

\section{Conclusion}

From the two derived Expressions (10) and (13) the values of means of usual closed conception interval and most recent closed conception interval for some hypothetical values of $T, \lambda, h$ and $i$ are obtained. As, both the expressions are functions of $T, \lambda, h$ and $i$, the behaviour of $E\left(X_{i}(T)\right)$ and $E\left(X_{i}^{l}(T)\right)$ is analysed by considering variations in all the above mentioned parameters. Table 8 presents the values of $\mathrm{E}\left(\mathrm{X}_{i}(T)\right)$ and $E\left(X_{i}^{l}(T)\right)$, for $T=60$ months, $\mathrm{h}=12$ months and for different values of $\lambda=0.04,0.06$ and 0.08 . Similar values are presented in Tables 9 and 10, for $T=90$ and 120 months respectively. Similarly, Table 11, presents about the variation in $E\left(X_{i}(T)\right)$ and $E\left(X_{i}^{l}(T)\right)$ by changing h (i.e. $\mathrm{h}=12,14$ and 16 months) keeping $\lambda=$ 0.06 and $T=60$ months. Of course, similar tables can be prepared for other combinations of values of $\lambda, T$, and $h$ also but we restrict our investigation to these tables only. From these tables, the following points emerge conclusively.

Table 8: Values of $E\left(X_{i}(T)\right)$ (in months), $E\left(X_{i}^{l}(T)\right)$ (in months)and Difference between $E\left(X_{i}(T)\right)$ and $E\left(X_{i}^{l}(T)\right)$ for $T=60$ months, $h=12$ months and different values of $\lambda(\lambda=0.04,0.06$ and 0.08$)$ 


\begin{tabular}{|c|l|l|l|l|l|l|l|l|l|}
\hline \multicolumn{3}{|c|}{$\lambda=0.04$} & \multicolumn{3}{c|}{$\lambda=0.06$} & \multicolumn{3}{c|}{$\lambda=0.08$} \\
\hline $\mathrm{i}$ & $E\left(X_{i}(T)\right)$ & $E\left(X_{i}^{l}(T)\right)$ & Difference & $E\left(X_{i}(T)\right)$ & $E\left(X_{i}^{l}(T)\right)$ & Difference & $E\left(X_{i}(T)\right)$ & $E\left(X_{i}^{l}(T)\right)$ & Difference \\
\hline 1 & 25.19 & 27.38 & 2.19 & 23.71 & 27.10 & 3.39 & 22.29 & 26.85 & 4.56 \\
2 & 20.28 & 20.65 & 0.38 & 19.86 & 20.49 & 0.63 & 19.42 & 20.33 & 0.91 \\
3 & 16.63 & 16.66 & 0.02 & 16.54 & 16.58 & 0.04 & 16.44 & 16.50 & 0.06 \\
4 & 13.98 & 13.98 & 0.00 & 13.96 & 13.96 & 0.00 & 13.95 & 13.95 & 0.00 \\
\hline
\end{tabular}

Table 9: Values of $E\left(X_{i}(T)\right)$ (in months), $E\left(X_{i}^{l}(T)\right)$ (in months) and Difference between $E\left(X_{i}(T)\right)$ and $E\left(X_{i}^{l}(T)\right)$ for $T=90$ months, $h=12$ months and different values of $\lambda(\lambda=0.04,0.06$ and 0.08$)$

\begin{tabular}{|c|l|l|l|l|l|l|l|l|l|}
\hline \multicolumn{3}{|c|}{$\lambda=0.04$} & \multicolumn{3}{c|}{$\lambda=0.06$} & \multicolumn{3}{c|}{$\lambda=0.08$} \\
\hline $\mathrm{i}$ & $E\left(X_{i}(T)\right)$ & $E\left(X_{i}^{l}(T)\right)$ & Difference & $E\left(X_{i}(T)\right)$ & $E\left(X_{i}^{l}(T)\right)$ & Difference & $E\left(X_{i}(T)\right)$ & $E\left(X_{i}^{l}(T)\right)$ & Difference \\
\hline 1 & 30.43 & 37.30 & 6.87 & 26.88 & 37.00 & 10.12 & 24.02 & 36.74 & 12.72 \\
2 & 25.87 & 28.04 & 2.17 & 24.32 & 27.83 & 3.52 & 22.76 & 27.64 & 4.89 \\
3 & 21.87 & 22.50 & 0.63 & 21.29 & 22.35 & 1.06 & 20.65 & 22.22 & 1.57 \\
4 & 18.68 & 18.82 & 0.14 & 18.48 & 18.72 & 0.24 & 18.26 & 18.63 & 0.37 \\
5 & 16.18 & 16.20 & 0.01 & 16.13 & 16.15 & 0.02 & 16.06 & 16.10 & 0.04 \\
6 & 14.23 & 14.23 & 0.00 & 14.21 & 14.21 & 0.00 & 14.20 & 14.20 & 0.00 \\
\hline
\end{tabular}

Table 10: Values of $E\left(X_{i}(T)\right)$ (in months), $E\left(X_{i}^{l}(T)\right)$ (in months) and Difference between $\mathrm{E}\left(\mathrm{X}_{i}(T)\right)$ and $E\left(X_{i}^{l}(T)\right)$ for $T=120$ months, $h=12$ months and different values of $\lambda(\lambda=0.04,0.06$ and 0.08$)$

\begin{tabular}{|l|l|l|l|l|l|l|l|l|l|}
\hline \multicolumn{3}{|c|}{$\lambda=0.04$} & \multicolumn{3}{c|}{$\lambda=0.06$} & \multicolumn{3}{c|}{$\lambda=0.08$} \\
\hline $\mathrm{i}$ & $E\left(X_{i}(T)\right)$ & $E\left(X_{i}^{l}(T)\right)$ & Difference & $E\left(X_{i}(T)\right)$ & $E\left(X_{i}^{l}(T)\right)$ & Difference & $E\left(X_{i}(T)\right)$ & $E\left(X_{i}^{l}(T)\right)$ & Difference \\
\hline 1 & 33.66 & 47.27 & 13.60 & 28.12 & 46.96 & 18.84 & 24.42 & 46.69 & 22.27 \\
2 & 30.12 & 35.49 & 5.37 & 26.86 & 35.27 & 8.41 & 24.06 & 35.08 & 11.02 \\
3 & 26.35 & 28.43 & 2.09 & 24.75 & 28.27 & 3.52 & 23.08 & 28.12 & 5.04 \\
4 & 22.96 & 23.74 & 0.78 & 22.25 & 23.61 & 1.36 & 21.45 & 23.50 & 2.05 \\
5 & 20.13 & 20.39 & 0.26 & 19.83 & 20.30 & 0.46 & 19.49 & 20.21 & 0.72 \\
6 & 17.81 & 17.88 & 0.07 & 17.70 & 17.82 & 0.12 & 17.57 & 17.76 & 0.19 \\
7 & 15.93 & 15.94 & 0.01 & 15.89 & 15.91 & 0.02 & 15.84 & 15.87 & 0.03 \\
8 & 14.38 & 14.38 & 0.00 & 14.37 & 14.37 & 0.00 & 14.35 & 14.35 & 0.00 \\
9 & 13.09 & 13.09 & 0.00 & 13.08 & 13.08 & 0.00 & 13.08 & 13.08 & 0.00 \\
\hline
\end{tabular}

Table 11: Values of $E\left(X_{i}(T)\right)$ (in months), $E\left(X_{i}^{l}(T)\right)$ (in months) and Difference between $E\left(X_{i}(T)\right)$ and $\mathrm{E}\left(\mathrm{X}_{i}^{l}(T)\right)$ for $T=60$ months, $\lambda=60$ months and different values of $h(h=12,14$ and 16 months)

\begin{tabular}{|l|l|l|l|l|l|l|l|l|l|}
\hline \multicolumn{3}{|c|}{$h=12$ months } & \multicolumn{3}{c|}{$h=14$ months } & \multicolumn{3}{c|}{$h=16$ months } \\
\hline $\mathrm{i}$ & $E\left(X_{i}(T)\right)$ & $E\left(X_{i}^{l}(T)\right)$ & Difference & $E\left(X_{i}(T)\right)$ & $E\left(X_{i}^{l}(T)\right)$ & Difference & $E\left(X_{i}(T)\right)$ & $E\left(X_{i}^{l}(T)\right)$ & Difference \\
\hline 1 & 23.71 & 27.10 & 3.39 & 25.39 & 28.20 & 2.81 & 27.07 & 29.31 & 2.24 \\
2 & 19.86 & 20.49 & 0.63 & 21.11 & 21.43 & 0.32 & 22.33 & 22.44 & 0.11 \\
3 & 16.54 & 16.58 & 0.04 & 17.46 & 17.46 & 0.00 & 18.34 & 18.33 & 0.00 \\
4 & 13.96 & 13.96 & 0.00 & & & & & & \\
\hline
\end{tabular}

1. In all the cases, $E\left(X_{i}(T)\right)$ is smaller than $E\left(X_{i}^{l}(T)\right)$. However, for maximum possible value of $i$, both means are identically equal. This is because of the fact that in this situation, by definition, the usual closed conception interval is the same as the most recent closed conception interval. 
2. For fixed values of $\lambda, h$, and $T$, the difference between $E\left(X_{i}(T)\right)$ and $E\left(X_{i}^{l}(T)\right)$ decreases as $i$ increases. Of course, as noted above, the difference is zero for maximum possible value of $i$.

3. For large values of $T$ and smaller $i$, the mean of usual closed conception interval is almost the same as the mean of $X_{i}\left(i . e . \frac{1}{\lambda}+h\right)$. This is because of the fact that for such values of $i$ and $T, G_{i+1}(T)$ is almost equal to one and $E\left(X_{i}(T)\right)$ becomes equal to $E\left(X_{i}\right)$.

4. As the value of is increased, $E\left(X_{i}(T)\right)$ and $E\left(X_{i}^{l}(T)\right)$ both decrease, but the decrease in $E\left(X_{i}^{l}(T)\right)$ is relatively smaller in comparison to the decrease in $E\left(X_{i}(T)\right)$.

5. As the value of $\mathrm{T}$ is increased, there is increment in the values of both the means, but, $E\left(X_{i}^{l}(T)\right)$ increases more rapidly.

6. As the value of $h$ is increased and other parameters are fixed, there is increment in the value of both the means.

Till now, the results based on the computed values of $E\left(X_{i}(T)\right)$ and $E\left(X_{i}^{l}(T)\right)$ for different combinations of values of the parameters $\lambda, T$, and $h$ are demonstrated. Now the issue is investigated by comparing the p. $d$ f.'s of the two distributions, viz., $h_{i}(X, T)$ and $h_{i}^{l}(X, T)$ (see Expressions (9) and (11)). The difference in the two distributions mainly occurs due to two ratios $R_{1}(x)$ and $R_{2}(x)$, which are explained below.

$$
h_{i}(x, T)\left\{\begin{array}{l}
\frac{G_{i}(T-x)}{G_{i+1}(T)} f(x), \quad h<x<T-(i-1) h \\
0, \quad \text { otherwise, }
\end{array}\right.
$$

and

$$
h_{i}^{l}(x, T)\left\{\begin{array}{l}
\frac{P_{i}(T-x)}{P_{i+1}(T)} f(x), \quad h<x<T-i h \\
\frac{G_{i}(T-x)}{P_{i+1}(T)} f(x), \quad T-i h<x<T-(i-1) h \\
0, \quad \text { otherwise, }
\end{array}\right.
$$

These can be written as

$$
\begin{aligned}
& h_{i}(x, T)=R_{1}(x) f(x) \\
& h_{i}^{l}(x, T)=R_{2}(x) f(x),
\end{aligned}
$$

where

$$
R_{1}(x)\left\{\begin{array}{l}
\frac{G_{i}(T-x)}{G_{i+1}(T)}, \quad h<x<T-(i-1) h \\
0, \quad \text { otherwise }
\end{array}\right.
$$

and

$$
R_{2}(x)\left\{\begin{array}{l}
\frac{P_{i}(T-x)}{P_{i+1}(T)}, \quad h<x<T-i h \\
\frac{G_{i}(T-x)}{P_{i+1}(T)}, \quad T-i h<x<T-(i-1) h \\
0, \quad \text { otherwise, }
\end{array}\right.
$$


The range of $x$, for both the probability distributions or the ratios $R_{1}(x)$ and $R_{2}(x)$, is $(h, T-(i-1) h)$. The plots of $R_{1}(x)$ and $R_{2}(x)$ for some hypothetical values of $\lambda, T$, and $h$ are drawn (as considered in the tables). These plots are presented in Figures 1,2 and 3. $R_{1}(x)$ is of monotonically decreasing nature implying that apart from $f(\mathrm{x})$, it gives larger weights to smaller values of $x$ and smaller weights to larger values of $x$. However, the behaviour of $R_{2}(x)$ is not monotonic. For smaller values of $i$, the curve seems to be bell shaped, whereas for the larger values of $i$, the two ratios i.e. $R_{1}(x)$ and $R_{2}(x)$ are almost identical. For smaller $i$, the behaviour of $R_{2}(x)$ is such that it is giving, on an average, larger weights to the larger values of $x$ in comparison to $R_{1}(x)$, implying that the $E\left(X_{i}^{l}(T)\right)$ will be larger than the $E\left(X_{i}(T)\right)$ for smaller values of $i$. But, for larger values of $i$, both the means will be almost equal. Thus, these gures give us the clue that why the $E\left(X_{i}^{l}(T)\right)$ is larger than the $E\left(X_{i}(T)\right)$ for smaller values of $i$ and why for large values of $i$, both are almost equal. The gures also testify the remarks given by Sheps and Menken (1973).

That the distribution of usual closed conception interval is analogous to the distribution of usual CBI and similarly the distribution of most recent closed conception interval is analogous to the distribution of most recent CBI. The only difference is about the shift of origin in marital duration in the sense that conceptions in time $(0, T)$ are same as births in time $(g, g+T)$. Hence, both can be studied interchangeably by making suitable change in origin.

Above results are based on some simplifying assumptions such as closed conception intervals are identically distributed as displaced exponential distribution with parameters and $\mathrm{h}$. It is not sure that what will be the behaviour of $E\left(X_{i}(T)\right)$ and $E\left(X_{i}^{l}(T)\right)$, if other distributions for $f_{i}(x)$ are taken. However, these at least demonstrate that the most recent CBI is di erent from the usual CBI. Thus, more work is needed to study the relationship between the two under more realistic assumptions. It is also important to mention that the derived results are purely of theoretical nature, which do not account for any type of non-sampling error. However, it is a known fact that the birth interval data are prone to error of recall lapse and memory bias. In this context, the most recent $\mathrm{CBI}$ is likely to be more reliable than the other birth intervals. Thus, it is desirable to use the data of most recent CBI for studying fertility behaviour of females between two births, but, one should be cautious about accounting for the theoretical difference between the most recent $\mathrm{CBI}$ and the usual CBI. 
Figure 1: Plots of $R_{1}(x)$ and $R_{2}(x)$ for $T=60$ months, $\lambda=0.04, h=12$ months and $i=1,2,3$ and 4.

$\mathrm{i}=1$

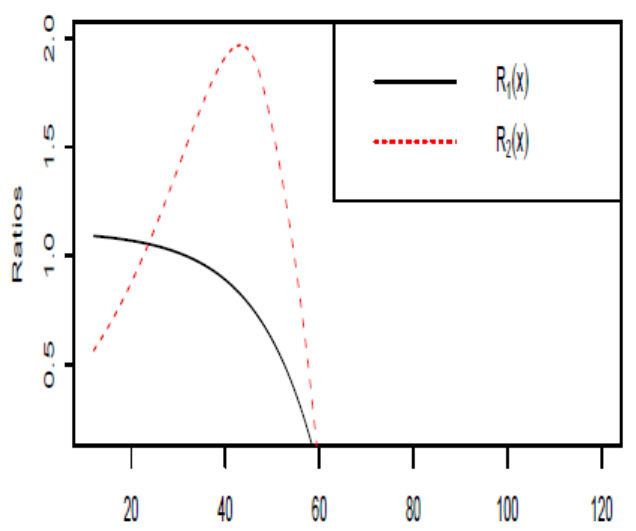

$x \rightarrow$

$j=3$

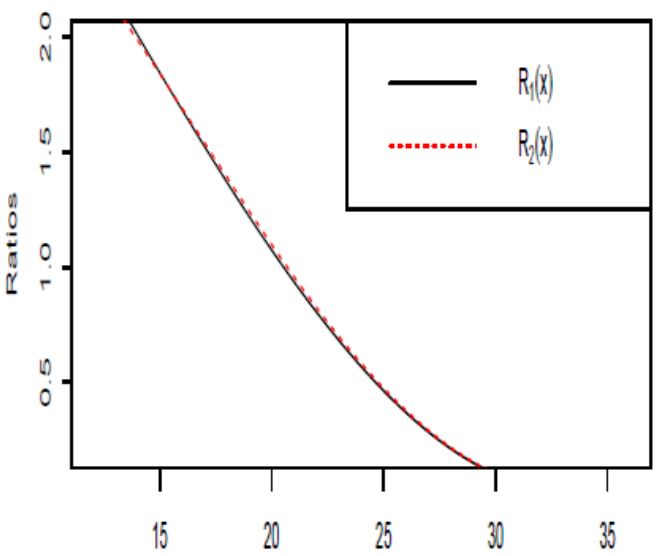

$x \rightarrow$. $i=2$

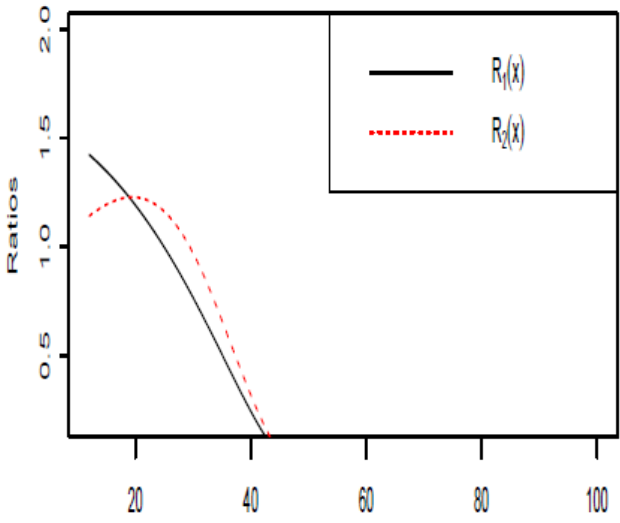

$x \rightarrow$.

$i=4$

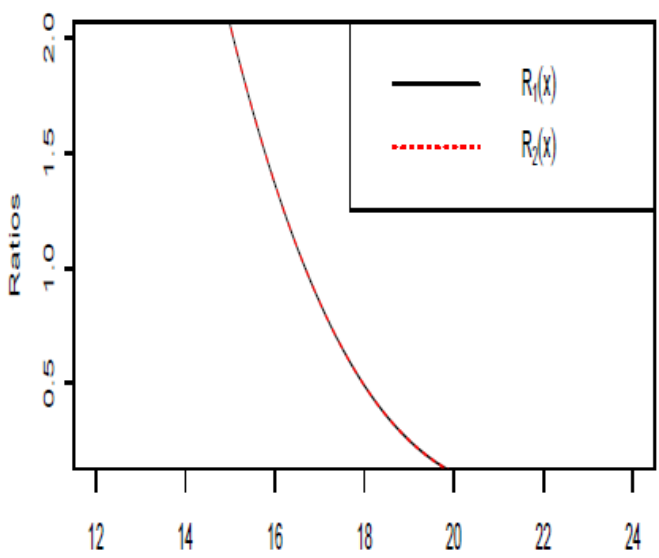

$x \rightarrow$ 
Figure 2: Plots of $R_{1}(x)$ and $R_{2}(x)$ for $T=90$ months, $\lambda=0.06, h=12$ months and different values of $i$.

$\mathrm{i}=1$

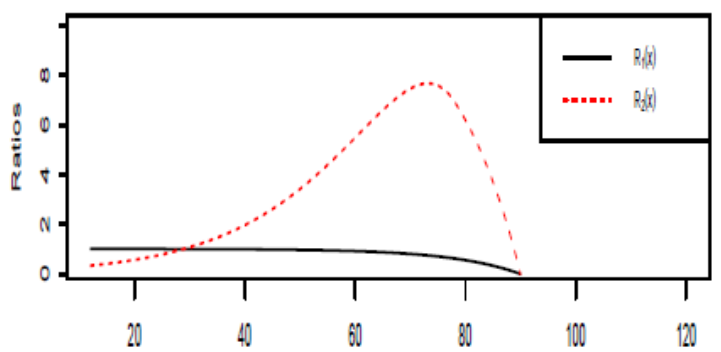

$x \rightarrow$

$i=3$

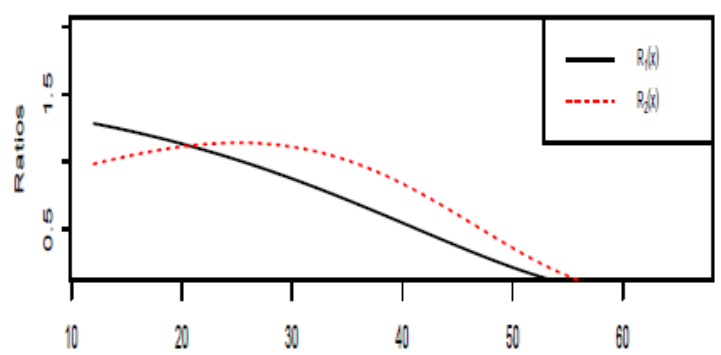

$x \rightarrow$

$j=5$

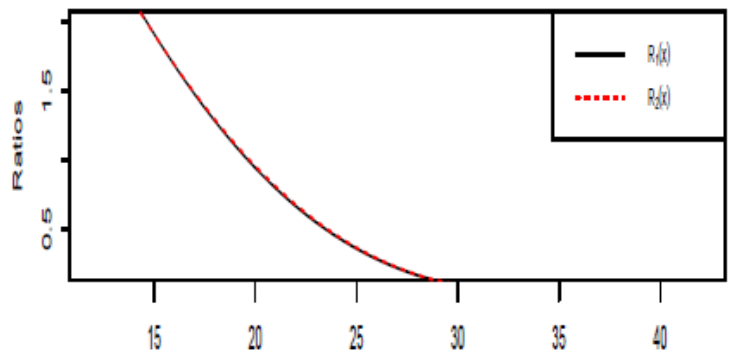

$i=2$

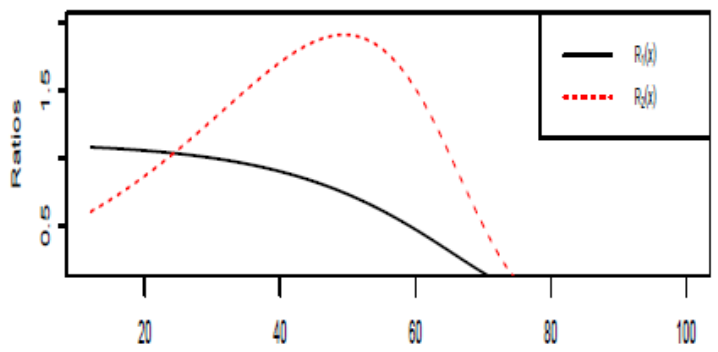

$x \rightarrow$

$i=4$

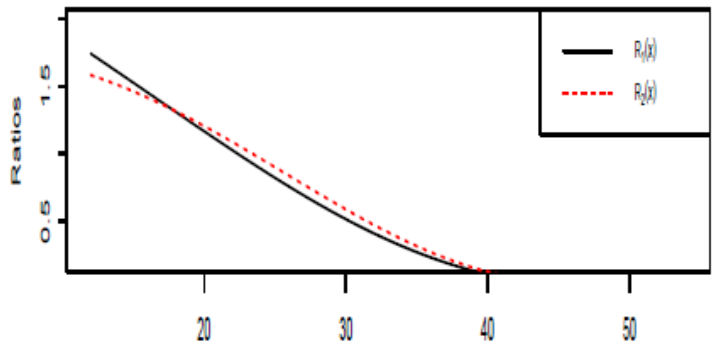

$x \rightarrow$.

$j=6$

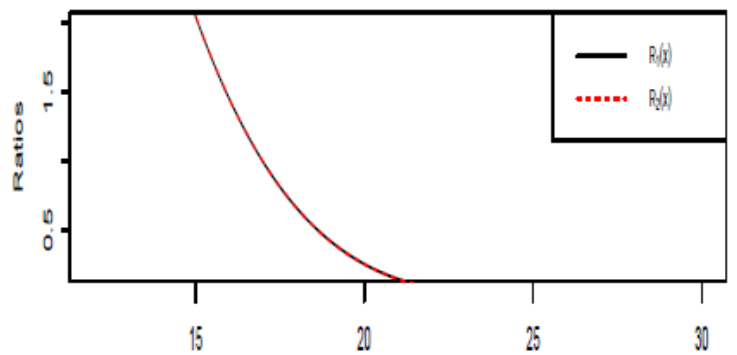

$x \rightarrow$ 
Figure 3: Plots of $R_{1}(x)$ and $R_{2}(x)$ for $T=120$ months, $\lambda=0.08, h=12$ months and different values of $i$.

$\mid=1$

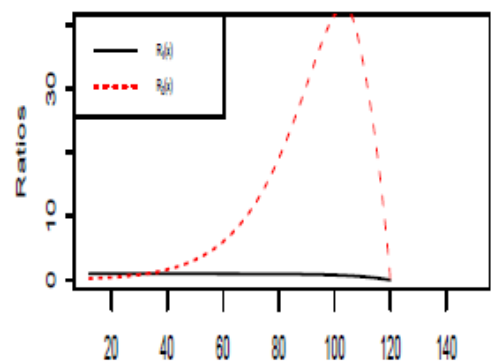

$x \rightarrow-$

$i=4$
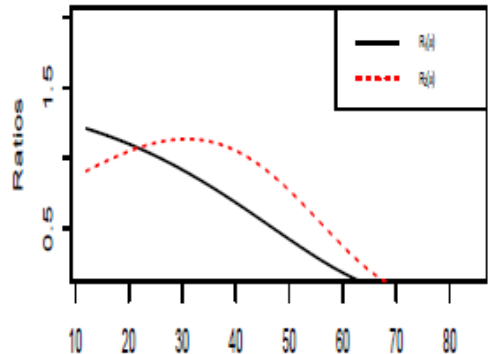

$x \rightarrow$.

$\mid=7$

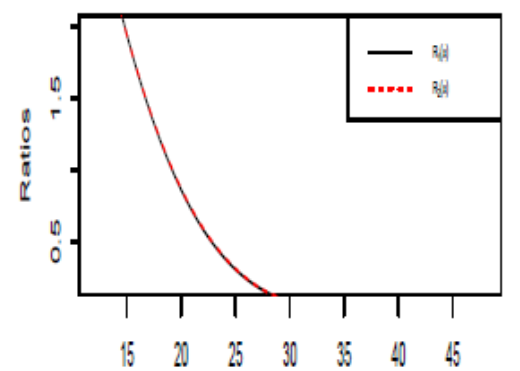

$x \rightarrow$ $i=2$

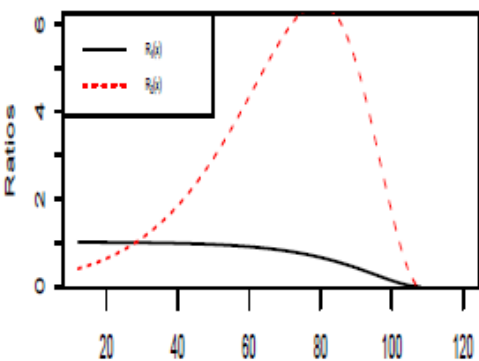

$x \rightarrow$.

$j=5$

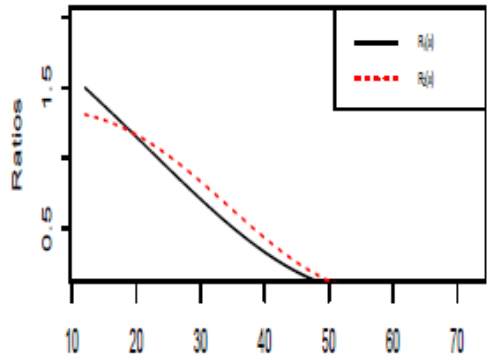

$x \rightarrow$

$\mid=8$

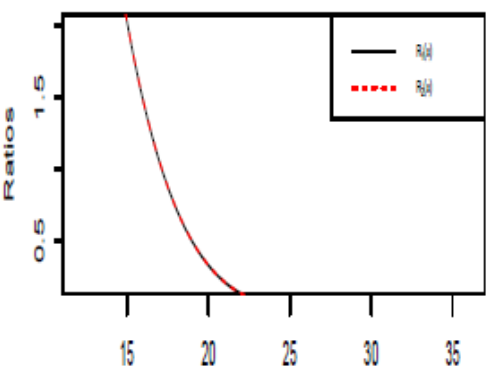

$x \rightarrow$ $\mid=3$

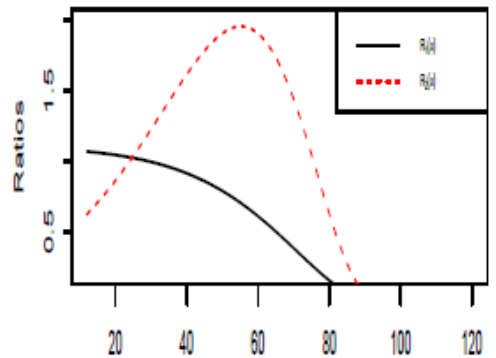

$x \rightarrow$.

$i=6$

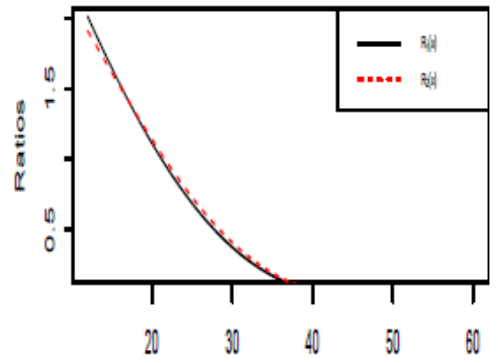

$x \rightarrow-$

$j=9$

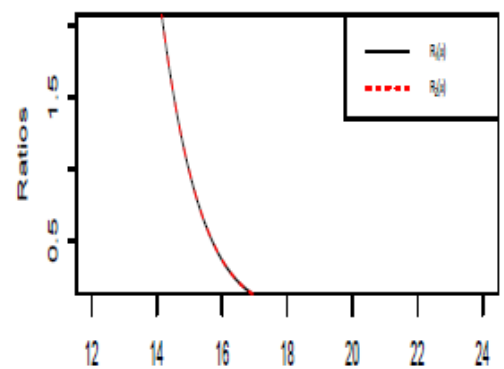

$x \rightarrow$. 


\section{References}

[1] Abul-ata, M. F. (1987). Stochastic Models of Birth Intervals According to data Ascertainment Method and Relevant Fertility Indices. $\mathrm{PhD}$ thesis, Department of Biostatistics, University of North Carolina at Chapel Hill, Institute of Statistics.

[2] Fisher, R. A. (1934). The effect of the methods of ascertainment upon the estimation of frequencies. Annals of Eugenics, 6:13-25.

[3] Pandey, A., Dwivedi, S. N., and Mishra, R. N. (1998). Distribution of last closed birth intervals with some bio-social components: A stochastic model and its applications. Mathematical Population Studies, 7 (1):1-27.

[4] Poole, K. (1973). Fertility measure based on birth interval data. Theoritical Population Biology, 4:357-387.

[5] Rao, C. R. (1965). On Discrete Distributions Arising Out of Method of Ascertainment, chapter Classical and contagious distributions, pages 320-332. Statistical publishing society, Calcutta.

[6] Sehgal, J. M. (1971). Indices of Fertility Derived from Data on the Length of Birth Intervals, Using Different Ascertainment Plans. PhD thesis, Department of Biostatistics, University of North Carolina at Chapel Hill, Institute of Statistics, Mimco Series 768.

[7] Sharma, S. S. (2004). A Study on Some Mathematical Models for Birth Intervals. PhD thesis, Banaras Hindu University.

[8] Sheps, M. C. and Menken, J. A. (1972). Distribution of birth interval according to the sampling frame. Theoritical Population Biology, 3:1-26.

[9] Sheps, M. C. and Menken, J. A. (1973). Mathematical Models of Conception and Birth. The University of Chicago Press, Chicago and London.

[10] Sheps, M. C., Menken, J. A., Ridley, J. C., and Linger, J. W. (1970). Truncation effect in closed and open birth interval data. Journal of American Statistical Association, 65(330):678-693.

[11] Singh, S. N. (1968). A chance mechanism of the variation in the number of births per couple. Journal of the American Statistical Association, 63(321):209-213.

[12] Singh, S. N., Bhattacharya, B. N., and Yadava, R. C. (1971). On some distribution of couple fertility and their applications. In Singh, S. N., editor, Proceedings of all India seminar Demography and Statistics, pages 76-94. Banaras Hindu University. 
[13] Singh, S. N., Bhattacharya, B. N., and Yadava, R. C. (1974). A parity dependent model for number of births and its applications. Sankhya, Ser B, 36(1):93-102.

[14] Singh, S. N., Bhattacharya, B. N., and Yadava, R. C. (1979). An adjustment of a selection bias in postpartum amenorrhea from follow- up studies. Journal of the American Statistical Association, 74:916-920.

[15] Singh, U. P., Singh, V. K., and Singh, O. P. (1988). A parity dependent model for the most recent birth interval. Canadian studies in population, 15 (1):25-30.

[16] Srinivasan, K. (1967). Analytical Models for Two Types of Birth Intervals With Applications to Indian Population. PhD thesis, University of Kerala, Trivandrum, India.

[17] Srinivasan, K. (1980). Birth interval analysis in fertility surveys. Technical report, WFS, Scientific Reports, No. 7. London: World Fertility Survey.

[18] Wolfers, D. (1968). Determinants of birth intervals and their means. Population Studies, 22:253-262.

[19] Yadava, R. C. and Sharma, S. S. (2004). Closed birth interval verses most recent closed birth interval. Demography India, 33(2):249-263.

Received December 12, 2013; accepted July 26, 2014.

Anup Kumar

Department of Statistics, University of Allahabad

Allahabad, India - 211002

R. C. Yadava

Department of Statistics \& DST-CIMS, Banaras Hindu University

Varanasi, India - 221005 
